Ilchmann, Achim ; Owens, David H. :

Threshold switching functions in high-gain adaptive control

Zuerst erschienen in:

IMA Journal of Mathematical Control and Information 8 (1991), S. $409-429$ 


\title{
Threshold Switching Functions in High-Gain Adaptive Control
}

\author{
Achim Ilchmann and David H. Owens \\ Centre for Systems and Control Engineering, \\ School of Engineering, University of Exeter, \\ North Park Road, Exeter EX4 $4 Q F, U K$ \\ ilchmann. aQexeter.ac.uk
}

[Received 1 September 1991 and in revised form 25 November 1991]

Adaptive stabilizers for the class of $m$-input $m$-output minimum phase systems

$$
\dot{x}=A x+B u, \quad y=C x
$$

with unknown state dimension and definite high-frequency gain, i.e. $\sigma(C B) \subset$ $\mathbb{C}_{-}$or $\sigma(C B) \subset \mathbb{C}_{+}$, are introduced. These controllers are not based on identification mechanisms and yield an exponential decaying output and a convergent adaptation gain. The main result is a switching strategy alternative to the so-called Nussbaum function approach. This adaptation mechanism can tolerate dynamics in the input and, more importantly, sector-bounded input-output nonlinearities are allowed for single-input single-output systems.

\section{Nomenclature}

$\mathbb{R}_{+}\left(\mathbb{R}_{-}\right)$: the set of non-negative (non-positive) real numbers.

$\mathbb{C}_{+}\left(\mathbb{C}_{-}\right)$: the open right- (left-) half complex plane.

$\sigma(A)$ : the spectrum of the matrix $A \in \mathbb{C}^{n \times n}$.

$\|x\|_{P}=\sqrt{\langle x, P x\rangle}$ for $x \in \mathbb{R}^{n}, P=P^{T} \in \mathbb{R}^{n \times n}$ positive.

$L_{p}(J)$ : vector space of measurable functions $f: J \rightarrow \mathbb{R}^{n}, J \subset \mathbb{R}$ some interval and $n$ being defined by the context, such that $\|f(\cdot)\|_{L_{p}(J)}<\infty$, where

$$
\|f(\cdot)\|_{L_{p}(J)}:= \begin{cases}{\left[\int_{J}\|f(s)\|^{p} d s\right]^{1 / p}} & \text { for } \mathrm{p} \in[1, \infty) \\ \operatorname{ess~sup}_{\mathrm{s} \in \mathrm{J}}\|\mathrm{f}(\mathrm{s})\| & \text { for } \mathrm{p}=\infty\end{cases}
$$

\section{Introduction}

THE PROBLEM of high-gain adaptive stabilization of uncertain systems has been studied by various authors, see, for example, Willems \& Byrnes (1984), Byrnes \& Willems (1984), Mårtensson (1986), Morse (1987), Ilchmann et al. (1987), Miller \& Davison (1989). In general terms, this problem can be regarded as the construction of an output feedback mechanism driven by a gain adaptation depending nonlinearly 
on the output and capable of stabilizing any system within a class of systems defined in terms of structural properties only. In this paper, we study the problem of adaptive stabilization of multi-input multi-output, minimum-phase, relative-degreeone systems of the form

$$
\left.\begin{array}{l}
\dot{x}(t)=A x(t)+B u(t), \quad x(0) \in \mathbb{R}^{n} \\
y(t)=C x(t)
\end{array}\right\}
$$

More precisely, we study the class $\Sigma$ consisting of systems $(A, B, C) \in \mathbb{R}^{n \times n} \times$ $\mathbb{R}^{n \times m} \times \mathbb{R}^{m \times n}$, associated with (1.1), which satisfy the minimum-phase condition

$$
\operatorname{det}\left[\begin{array}{cc}
s I-A & B \\
C & 0
\end{array}\right] \neq 0 \quad \text { for all } \mathrm{s} \in \overline{\mathbb{C}}_{+}
$$

and which have a high-frequency gain of definite sign, i.e. the spectrum fulfils

$$
\sigma(C B) \subset \mathbb{C}_{-} \quad \text { or } \quad \sigma(C B) \subset \mathbb{C}_{+}
$$

Note that neither $(A, B, C)$ nor the state dimension $n$ need be known.

It is well known that such systems can be stabilized by a time-varying output feedback containing gain adaptation and a Nussbaum-based switching mechanism. An example of this (see, for example, Nussbaum 1983, Byrnes \& Willems 1984) is the feedback

$$
u(t)=N(k(t)) k(t) y(t)
$$

with

$$
\dot{k}(t)=\|y(t)\|^{2}, \quad k(0)=k_{0} \in \mathbb{R}
$$

where $N(\cdot): \mathbb{R} \rightarrow \mathbb{R}$ is a Nussbaum function, i.e. a piecewise right continuous function which satisfies the so-called Nussbaum condition

$$
\sup _{k>0} \frac{1}{k} \int_{0}^{k} N(\tau) \tau d \tau=+\infty \quad \text { and } \quad \inf _{k>0} \frac{1}{k} \int_{0}^{k} N(\tau) \tau d \tau=-\infty
$$

Examples of Nussbaum functions are $N(q)=q \sin \sqrt{q}$ or

$$
N(q)=\left\{\begin{array}{cll}
1 & , n^{2} \leq|q|<(n+1)^{2}, & n \text { even } \\
-1 & , n^{2} \leq|q|<(n+1)^{2}, & n \text { odd }
\end{array}\right.
$$

Note that the switching times in this approach depend solely on the instantaneous value of the gain $k(t)$ and, in general, contribute to the total gain $N(k) k$ at any time and hence to the limit gain.

Alternatively, the feedback law

$$
u(t)=N(s(t)) k(t) y(t)
$$

where $N(q) / q$ is a Nussbaum function, permits a wider class of gain evolutions, see Owens et al. (1987), with switching dependent on the instantaneous value of $s(t)$ rather than $k(t)$ but the total gain in this case is affected by the switching strategy. 
These switching functions are replaced in this paper by a new class of switching mechanisms where switching times are decoupled from the magnitude of the instantaneous gain and make no contribution to the total control gain in the way that previous approaches require. The switching mechanisms have all the advantages of those previously reported but avoid the difficulties described above.

Together with the switching strategy, in this paper we also introduce classes of adaptive control laws that can tolerate certain state, input and output nonlinearities of the following form.

Let the functions

$$
\begin{array}{ll}
g: & \mathbb{R}_{+} \times \mathbb{R}^{n} \rightarrow \mathbb{R}^{n}, \quad(t, x) \mapsto g(t, x) \\
& \|g(t, x)\| \leq \hat{g}\|x\| \quad \forall(t, x) \in \mathbb{R}_{+} \times \mathbb{R}^{n} \\
h: & \mathbb{R}_{+} \times \mathbb{R}^{n} \rightarrow \mathbb{R}^{n}, \quad(t, x) \rightarrow h(t, x) \\
& \|h(t, x)\| \leq \hat{h}\|x\| \quad \forall(t, x) \in \mathbb{R}_{+} \times \mathbb{R}^{n}
\end{array}
$$

be measurable in $t$ for each fixed $x$ and locally Lipschitz in $x$ for each fixed $t$, and of finite gain, i.e. (1.8), (1.9) are satisfied for some (unknown) $\hat{g}, \hat{h} \geq 0$. These functions are build into the system (1.1) as follows

$$
\left.\begin{array}{l}
\dot{x}(t)=A x(t)+g(t, x(t))+B[u(t)+h(t, x(t))], \quad x(0) \in \mathbb{R}^{n} \\
y(t)=C x(t)
\end{array}\right\}
$$

The possibility of including $g(\cdot, \cdot)$ into the system equation, and showing that all propositions are valid if the linear bound $\hat{g}$ is small enough, demonstrates that the problems are well posed. This, together with the nonlinear disturbance $h(\cdot, \cdot)$, of bounded gain in the input, shows that the system is robust with respect to some unmodelled system (and sensor) dynamics.

In the single-input single-output case, the adaptive stabilizers tolerate the following large class sector-bounded nonlinearities.

Let $\beta \geq \alpha>0$. The set of sector-bounded functions $\mathcal{S B}(\alpha, \beta)$ consists of all functions

$$
f: \mathbb{R}_{+} \times \mathbb{R}, \quad(t, v) \mapsto f(t, v)
$$

which satisfy, for all $t \in \mathbb{R}_{+}$,

$$
\left.\begin{array}{ll}
\alpha v \leq f(t, v) \leq \beta v & \forall v \in \mathbb{R}_{+} \\
\alpha v \geq f(t, v) \geq \beta v & \forall v \in \mathbb{R}_{-}
\end{array}\right\}
$$

For $\xi, \eta \in \mathcal{S B}(\alpha, \beta)$, we consider the system

$$
\begin{aligned}
& \dot{x}(t)=A x(t)+g(t, x(t))+B[u(t)+h(t, x(t))], \quad x(0) \in \mathbb{R}^{n} \\
& y(t()=C x(t) \\
& u(t)=\xi(t, \hat{u}(t)), \quad y(t)=\eta(t, y(t))
\end{aligned}
$$

illustrated in the following Figure 1. 

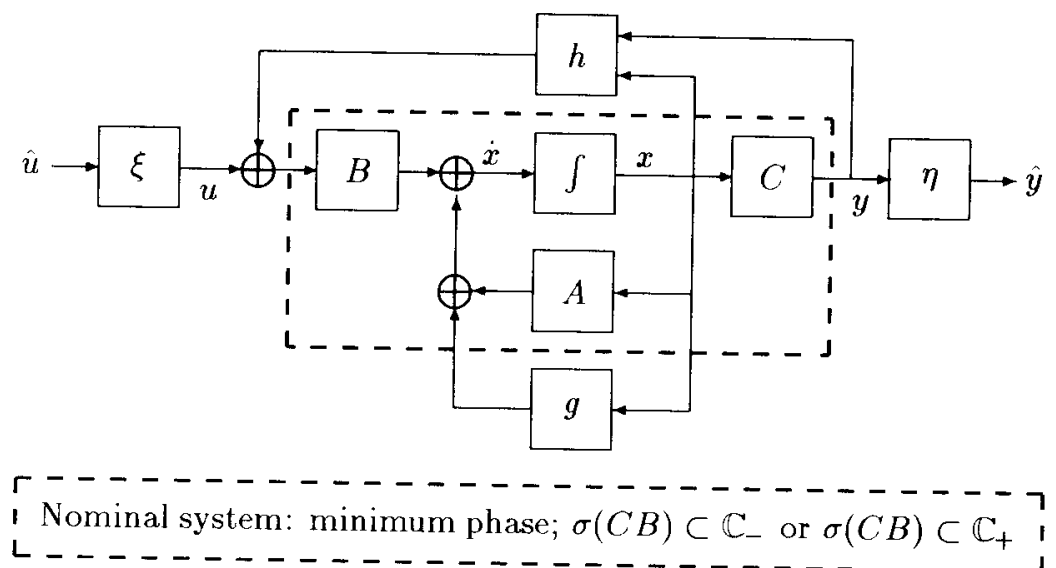

FIG.1 Open-loop system with nonlinearities

As mentioned above, a primary purpose of this paper is to introduce a new class of bounded threshold switching operations generalizing that introduced in Ilchmann and Owens (1991). Together with the advantages previously mentioned, this switching strategy is able to cope with nonlinear perturbations of the state space equations (as described above) and a large class of nonlinear input-output characteristics.

A second contribution of the paper is the use of a multi-input multi-output $L_{p}$, $p \geq 1$, formulation of the stability analysis that extends the previously available gain adaptation mechanisms. Finally, the paper allows the use of exponential weightings in the manner of Ilchmann and Owens (1990) to permit the development of exponentially stabilizing feedback schemes.

In Section 2, some properties of the class of systems under consideration are stated. The main result is given in Section 3 , that is, a switching function based on a switching decision function and an exponentially weighted gain adaptation which yield a universal adaptive stabilizer in the presence of nonlinearities. This result is extended in Section 4, where it is shown that the adaptive stabilizer can tolerate sector-bounded nonlinearities in the input and output if the system class is restricted to single-input single-output systems. Finally, some simulations are presented in Section 5 .

\section{The system class}

In this section we establish fundamental properties of the class $\Sigma$, that is, systems of the form (1.1) which satisfy (1.2) and (1.3).

The following lemma shows a convenient form into which every system with $\operatorname{det}(C B) \neq 0$ can be converted by a suitable state space transformation.

LEMMA 2.1. Consider the system

$$
\left.\begin{array}{l}
\dot{x}(t)=A x(t)+B u(t), \quad x\left(t_{0}\right)=x_{0} \in \mathbb{R}^{n} \\
y(t)=C x(t)
\end{array}\right\}
$$

with $(A, B, C) \in \mathbb{R}^{n \times n} \times \mathbb{R}^{n \times m} \times \mathbb{R}^{m \times n}$. 
(i) If $\operatorname{det}(C B) \neq 0$, then the coordinate transformation $S^{-1} x=\left[y^{T}, z^{T}\right]^{T}$, where $S=\left[B(C B)^{-1}, V\right] \in G l_{n}(\mathbb{R})$ and $V \in \mathbb{R}^{n \times(n-m)}$ is a basis matrix of ker $C$, converts the system (2.1) into the form

$$
\left.\begin{array}{l}
\dot{y}(t)=A_{1} y(t)+A_{2} z(t)+C B u(t) \\
\dot{z}(t)=A_{3} y(t)+A_{4} z(t)
\end{array}\right\}
$$

where $A_{1}, \ldots, A_{4}$ are real matrices of appropriate formats.

(ii) If $(A, B, C) \in \mathrm{Z}$, then $A_{4}$ in (2.2) is asymptotically stable, that is, $\sigma\left(A_{4}\right) \subset \mathbb{C}_{-}$.

The proof of Lemma 2.1 is straightforward and is omitted for brevity.

In order to include the possibility of exponential stabilization in the manner of Ilchrnann and Owens (1990), we introduce the following notation:

Let $0 \leq t_{0}<t^{\prime} \leq \infty, \lambda(\cdot): \mathbb{R} \rightarrow \mathbb{R}^{r}$ be continuously differentiable and $v(\cdot)$ : $\mathbb{R} \rightarrow \mathbb{R}^{r}, r \in \mathbb{N}$, be a vector-valued function. Then $v_{\lambda}(\cdot)$ will be defined by

$$
v_{\lambda}(t):=e^{\lambda(t) t} v(t)
$$

REMARK 2.2. Suppose $\lambda(\cdot): \mathbb{R}_{+} \rightarrow \mathbb{R}_{+}$is continuously differentiable. Consider (1.10) where it is assumed that $(A, B, C) \in \Sigma$ is subjected to the perturbations (1.8) and (1.9). Then, by Lemma 2.1, there exists a state space transformation $S \in \mathbb{R}^{n \times n}$ such that $\left[y^{T}, z^{T}\right]^{T}=S^{-1} x$ and the new coordinates $y_{\lambda}, z_{\lambda}$ satisfy

$$
\begin{array}{r}
\dot{y}_{\lambda}(t)=\left[A_{1}+(\lambda(t)+\dot{\lambda}(t) t) I_{m}\right] y_{\lambda}(t)+A_{2} z_{\lambda}(t)+C B u_{\lambda}(t) \\
+g^{1}\left(t, y_{\lambda}(t), z_{\lambda}(t)\right)+C B \bar{h}\left(t, y_{\lambda}(t), z_{\lambda}(t)\right) \\
\dot{z}_{\lambda}(t)=A_{3} y_{\lambda}(t)+\left[A_{4}+(\lambda(t)+\dot{\lambda}(t) t) I_{n-m}\right] z_{\lambda}(t)+g^{2}\left(t, y_{\lambda}(t), z_{\lambda}(t)\right)
\end{array}
$$

where $\sigma\left(A_{4}\right) \subset \mathbb{C}_{-}$, and $g^{1}(t, y, z) \in \mathbb{R}^{m}, g^{2}(t, y, z) \in \mathbb{R}^{n-m}$ are such that

$$
\left.\begin{array}{l}
\left\|g^{i}(t, y, z,)\right\| \leq \hat{g}\|S\| \|\left[y^{T}, z^{T}\right]^{T} \\
\|\tilde{h}(t, y, z)\| \leq h\|S\|\left\|\left[y^{T}, z^{T}\right]^{T}\right\|
\end{array}\right\} \quad \forall(t, y, z) \in \mathbb{R}_{+} \times \mathbb{R}^{m} \times \mathbb{R}^{n-m},
$$

for $\hat{g}, \hat{h}$ given in (1.8), (1.9) and $i=1,2$.

The following inequality bounds the output of the system (1.10) at time $t$ in terms of $\left.u(\cdot)\right|_{[0, t)}$ and $\left.y(\cdot)\right|_{[0, t)}$. This is an important implication of the minimum-phase property and is fundamental to the proof of the main results of the paper.

Proposition 2.3. Let $P \in \mathbb{R}^{m \times m}$ be positive definite, $0 \leq t_{0}<t^{\prime} \leq \infty, p \geq 1$. Suppose that $u(\cdot):\left[0, t^{\prime}\right) \rightarrow \mathbb{R}^{m}$ is continuous and $\lambda(\cdot):\left[0, t^{\prime}\right) \rightarrow \mathbb{R}$ is continuously differentiable and satisfies $\lim _{t \rightarrow t^{\prime}} \lambda(t)=0$. Define

$$
\beta: \mathbb{R}^{m} \rightarrow \mathbb{R}^{m}, y \mapsto \beta(y)=\left\{\begin{array}{cc}
\frac{y}{\|y\|_{P}} & , y \neq 0 \\
0 & , y=0
\end{array}\right.
$$

Then the system $(A, B, C) \in \Sigma$ subjected to the disturbances (1.8),(1.9) in the form (1.10) satisfies for $\hat{g}>0$ sufficiently small, suitable $M=M\left(x_{0}\right)>0$ and for all $t \in\left[t_{0}, t^{\prime}\right)$

$$
\frac{1}{p}\left\|y_{\lambda}(t)\right\|_{P}^{p} \leq M+M \int_{t_{0}}^{t}\left\|y_{\lambda}(s)\right\|_{P}^{p} d s+\int_{t_{0}}^{t}\left\|y_{\lambda}(s)\right\|_{P}^{p-1}\left\langle\beta\left(y_{\lambda}(s)\right), P C B u_{\lambda}(s)\right\rangle d s
$$


Proof. The proof is an extension of the single-input single-output case presented in Ilchmann and Owens (1991a) and of the multivariable case for $g \equiv 0$ in Ilchmann and Logemann (1991). We omit it for brevity.

\section{Adaptive stabilization using threshold switching}

In order to use the gain adaptation class of exponential weightings similar to those defined in Ilchmann and Owens (1990) which ensures exponential decay of the output we introduce the following conventions.

Let $\omega(\cdot): \mathbb{R}_{+} \rightarrow \mathbb{R}_{+}$be a continuously differentiable function which satisfies the conditions

$$
\left.\begin{array}{l}
\omega(k) \text { is non-increasing in } k \in \mathbb{R}_{+} \\
\omega(k)>0 \text { for all } k \in \mathbb{R}_{+} \text {if } \omega(\cdot) \not \equiv 0 \\
\lim _{k \rightarrow \infty} \omega(k)=0
\end{array}\right\}
$$

Suppose $f: \mathbb{R}_{+} \times \mathbb{R}^{2+m} \rightarrow \mathbb{R}$ is a Carathéodory function such that for every absolutely continuous $y:\left[0, t^{\prime}\right) \rightarrow \mathbb{R}^{m}, t^{\prime} \in(0, \infty]$, there exists a unique absolutely continuous solution $k(\cdot):\left[0, t^{\prime}\right) \rightarrow \mathbb{R}_{+}$of

$$
\dot{k}(t)=f(t,(\omega \circ k)(t), y(t)), \quad k(0)=k_{0} \in \mathbb{R}
$$

so that

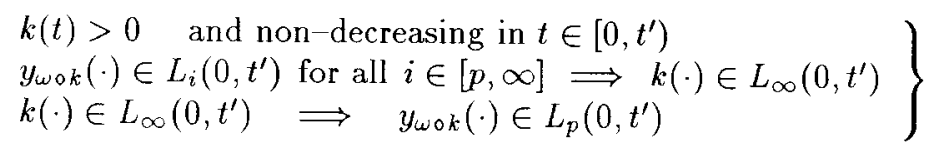

A simple example of $\omega(\cdot)$ and $k(\cdot)$ satisfying (3.1)-(3.3) is, for $p^{\prime} \geq p \geq 1$,

$$
\omega(k)=\frac{1}{k+1}, \quad \dot{k}(t)=\sum_{i=p}^{p^{\prime}}\left\|y_{\omega \circ k}(t)\right\|^{i}, \quad k(0)>-1
$$

Given $\omega(\cdot)$ and $k(\cdot)$ satisfying (3.1)-(3.3), the adaptive control is completely defined by

$$
u(i)=S(t) k(t) y(t)
$$

once the switching function $S(t)$ is defined. This will be done in the next definition.

Definition. Suppose $p \geq 1, \omega(\cdot)$ satisfies $(3.1)$, and $y(\cdot): \mathbb{R}_{+} \rightarrow \mathbb{R}^{m}$ is a $p$ integrable function. Let $1<S_{1}<S_{2}<\ldots$ be a sequence of 'thresholds' so that $\lim _{i \rightarrow \infty} S_{i}=\infty$. Let $k(\cdot): \mathbb{R}_{+} \rightarrow \mathbb{R}$ be a continuous and non-decreasing function with $k(0)>0$. Then the switching function

$$
S(\cdot): \mathbb{R}_{+} \rightarrow\{-1,1\}
$$

is defined via the switching decision function

$$
\varphi(t):= \begin{cases}k(0), & \text { if } \int_{0}^{t}\|y(\tau)\|^{p} d \tau=0 \\ \int_{0}^{t} S(\tau) k(\tau)\left\|y_{\omega \circ k}(\tau)\right\|^{p} d \tau / \int_{0}^{t}\left\|y_{\omega \circ k}(\tau)\right\|^{p} d \tau, & \text { if } \int_{0}^{t}\|y(\tau)\|^{p} d \tau \neq 0\end{cases}
$$


and the real-time algorithm

$$
\left.\begin{array}{l}
i:=0, \quad, t_{0}:=0, \quad S(0):=1 \\
t_{i+1}:=\inf \left\{t>t_{i} \mid S\left(t_{i}\right) \varphi(t)=S_{i+1} k\left(t_{0}\right)\right\} \\
S(t):=S\left(t_{i}\right) \text { for all } t \in\left[t_{i}, t_{i+1}\right) \\
S\left(t_{i+1}\right):=-S\left(t_{i}\right) \\
i:=i+1 \\
\text { go to }(*)
\end{array}\right\}
$$

REMARK 3.1 .

(i) Note that $\varphi(0)=k(0)$ which ensures correct initialization of the algorithm.

(ii) $\varphi(t)$ is monotonic on every interval where $S(\cdot)$ is constant.

(iii) In the case of $p=2$, the above algorithm is similar to that of Ilchmann and Owens (1991).

(iv) The switching function $S(\cdot)$ switches at each time $t_{i}$ if the switching decision function, which is a stability indicator, reaches the new 'threshold' $S_{i+1} k\left(t_{0}\right)$.

Stability of the adaptive scheme requires stability of the state, convergence of the gain adaptation and the presence of only a finite number of sign changes in $S(t)$. The proof of stability requires the analysis of the situation if the final sign of the switching function is incorrect, i.e. $S(t)=\operatorname{sgn}(C B)$. This is discussed in the following lemma. We use the following notation

$$
\operatorname{sgn}(C B):= \begin{cases}+1 & \text { if } \sigma(C B) \subset \mathbb{C}_{+} \\ -1 & \text { if } \sigma(C B) \subset \mathbb{C}_{-}\end{cases}
$$

LEMMA 3.2. Suppose $p \geq 1, t^{\prime}>0, k(\cdot):\left[0, t^{\prime}\right) \rightarrow \mathbb{R}_{+}$is absolutely continuous, $\omega(\cdot)$ satisfies (3.1), and the feedback law

$$
u(t)=\operatorname{sgn}(C B) k(t) y(t)
$$

is applied to $(A, B, C) \in \Sigma$ subjected to the nonlinearities (1.8),(1.9). If one sets $\lambda=\omega \circ k$, then, by Remark 2.2, the closed-loop system can be written in the form

$$
\begin{array}{r}
\dot{y}_{\lambda}=\left[A_{1}+(\lambda+\dot{\lambda} t) I_{m}\right] y_{\lambda}+A_{2} z_{\lambda}+\operatorname{sgn}(C B) C B k y_{\lambda} \\
+g^{1}\left(t, y_{\lambda}, z_{\lambda}\right)+C B \tilde{h}\left(t, y_{\lambda}, z_{\lambda}\right) \\
\dot{z}_{\lambda}=A_{3} y_{\lambda}+\left[A_{4}+(\lambda+\dot{\lambda} t) I_{n-m}\right] z_{\lambda}+g^{2}\left(t, y_{\lambda}, z_{\lambda}\right)
\end{array}
$$

If $k(\cdot) \notin L_{\infty}\left(0, t^{\prime}\right)$ and the linear bound $\hat{g}$ is small enough, then for every $\epsilon>0$ there exists $t_{\epsilon} \in\left(0, t^{\prime}\right)$ such that

$$
\left\|z_{w 0 k}(t)\right\| \leq \epsilon\left\|y_{\omega \circ k}(t)\right\| \text { for all } t \in\left[t_{\epsilon}, t^{\prime}\right)
$$


Proof. Since $g, h, k$ are Carathéodory functions and $g, h$ are linearly bounded in $x$, it follows that $\left[0, t^{\prime}\right)$ is the maximal interval of existence of $(3.6)$. Now we proceed in several steps.

Step (i). Let $P, Q>0$ denote the unique solutions of

$$
\begin{gathered}
(C B)^{T} P+P C B=\operatorname{sgn}(C B) I_{m} \\
A_{4}^{T} Q+Q A_{4}=-I_{n-m}
\end{gathered}
$$

Step (ii). Let $S$ be given as in Lemma 2.1 and $R=\operatorname{diag}(P, Q)$. Then we want to show that the function

$$
V(t)=\frac{\left\|y_{\lambda}(t)\right\|_{P}}{\left\|S^{-1} x_{\lambda}(t)\right\|_{R}} \in[0,1]
$$

has the property

$$
\lim _{t \rightarrow t^{\prime}} V(t)=1
$$

It then would follow that for any $\epsilon^{\prime}>0$ there exists $t_{\epsilon} \in\left(0, t^{\prime}\right)$ such that for all $t \in\left[t_{\epsilon}, t^{\prime}\right)$, we have $\left\|y_{\lambda}\right\|_{P}^{2} \geq\left(1-\epsilon^{\prime}\right)\left\|S^{-1} x_{\lambda}\right\|_{R}^{2}=\left(1-\epsilon^{\prime}\right)\left(\left\|z_{\lambda}\right\|_{Q}^{2}+\left\|y_{\lambda}\right\|_{P}^{2}\right)$. Now a simple rearrangement yields (3.7).

Step (iii). The set

$$
J:=\left\{t \in\left[0, t^{\prime}\right) \mid ; x(t) \text { is not differentiable or }[x(t)=0 \text { and } \dot{x}(t) \neq 0]\right\}
$$

is of measure zero, see Ilchmann and Logemann (1991).

Step (iv). Differentiation at $t \in\left[0, t^{\prime}\right) \backslash J$ yields

$$
\begin{aligned}
\frac{d}{d t}\left(\left\|S^{-1} x_{\lambda}(t)\right\|_{R}^{-1}\right) & =-\frac{\left\langle S^{-1} x_{\lambda}(t), R S^{-1} \dot{x}_{\lambda}(t)\right\rangle}{\left\|S^{-1} x_{\lambda}(t)\right\|_{R}^{3}} \\
& =-\frac{\left\langle y_{\lambda}(t), P \dot{y}_{\lambda}(t)\right\rangle+\left\langle z_{\lambda}(t), Q \dot{z}_{\lambda}(t)\right\rangle}{\left\|S^{-1} x_{\lambda}(t)\right\|_{R}^{3}} \\
\frac{d}{d t}\left(\left\|y_{\lambda}(t)\right\|_{P}\right) & =\frac{\left\langle y_{\lambda}(t), P \dot{y}_{\lambda}(t)\right\rangle}{\left\|y_{\lambda}(t)\right\| P}
\end{aligned}
$$

We simplify the notation by introducing

$$
\hat{y}(t):=\left\|y_{\lambda}(t)\right\|_{P}, \quad \hat{z}(t):=\left\|z_{\lambda}(t)\right\|_{Q}, \quad x(t):=\left\|S^{-1} x_{\lambda}(t)\right\|_{R}
$$

Step (v). Differentiation of $V(t)$ at $t \in\left[0, t^{\prime}\right) \backslash J$ along (3.6) and using (iv) yields, omitting the argument $t$,

$$
\begin{gathered}
\dot{V}=\frac{\dot{z}^{2}}{x^{3} y}\left\langle y_{\lambda}, P A_{1} y_{\lambda}+P A_{2} z_{\lambda}+\operatorname{sgn}(C B) k P C B y_{\lambda}+P\left[g^{1}+C B \tilde{h}\right]\right\rangle \\
-\frac{\hat{y} \hat{z}}{x^{3}}\left\langle z_{\lambda}, Q A_{3} y_{\lambda}+Q A_{4} z_{\lambda}+Q g^{2}\right\rangle
\end{gathered}
$$

Note that the coefficients of $(\lambda+\dot{\lambda} t)$ cancel. Using the linear bounds of $g, h$ and (3.8), (3.9) we obtain the following lower bound for some $M>0$

$$
\begin{gathered}
\dot{V} \geq-M \frac{\dot{z}^{2}}{x^{3} \hat{y}}\left[\dot{y}^{2}+\dot{y} z+\hat{y}(\hat{z}+\hat{y})\right]-M \frac{\hat{y}}{x^{3}}[\hat{z}(\hat{z}+\hat{y})+\hat{y} z]+\frac{1}{2}(k+1) \frac{\hat{y} z^{2}}{x^{3}} \\
-3 M \frac{\hat{z}^{2} \hat{y}}{x^{3}}-2 M \frac{\hat{z}^{3}}{x^{3}}-2 M \frac{\hat{z} \hat{y}^{2}}{x^{3}}+\frac{1}{2}(k+1) \frac{\hat{y} \hat{z}^{2}}{x^{3}}
\end{gathered}
$$


Step (vi). Consider first all points $t \in\left[0, t^{\prime}\right)$ such that

$$
V(t) \in[\delta, 1-\delta] \text { where } 0<\delta<1 / 2
$$

Then it follows that

$$
\underline{\delta} \hat{y}<\hat{z}<\bar{\delta} \hat{y} \quad \text { where } \quad \underline{\delta}:=\sqrt{\delta} \sqrt{\frac{2-\delta}{1-\delta}}, \quad \bar{\delta}:=\frac{\sqrt{1-\delta^{2}}}{\delta}
$$

Therefore we conclude from (3.12) that for all $t \in\left\{\left[0, t^{\prime}\right) \backslash J \mid V(t) \in[\delta, 1-\delta]\right\}$

$$
\dot{V}(t)>-7 M \bar{\delta}^{3}+\underline{\delta}^{2} \frac{1}{2}(k(t)+1)(1-\delta)^{3} .
$$

Since $\lim _{t \rightarrow t^{\prime}} k(t)=\infty$ it follows that there exists some $t_{0} \in\left(0, t^{\prime}\right)$ such that

$$
\dot{V}(t)>0 \text { for all } t \in\left\{\left[t_{0}, t^{\prime}\right) \backslash J \mid V(t) \in[\delta, 1-\delta]\right\}
$$

Since $\delta$ is arbitrary small and $J$ is of measure zero, this implies $\lim _{t \rightarrow t^{\prime}} V(t) \in\{0,1\}$.

Step (vii). It remains to consider the case $\lim _{t \rightarrow t^{\prime}} V(t)=0$. If this holds true, then for every $\epsilon>0$ there exists $t^{*}=t^{*}(\epsilon) \in\left(0, t^{\prime}\right)$ such that $\left\|y_{\lambda}(t)\right\|_{P}^{2} \leq \epsilon\left\|x_{\lambda}(t)\right\|_{R}^{2}$ for all $t \in\left[t^{*}, t^{\prime}\right)$. Therefore for

$$
\epsilon^{\prime}:=\left(\frac{\epsilon}{1-\epsilon}\right)^{1 / 2} \frac{\sigma_{\max }(Q)}{\sigma_{\min }(P)}
$$

where $\sigma_{\max }(Q)\left(\sigma_{\min }(P)\right)$ denotes the maximal (minimal) singular value of $Q(P)$, we obtain

$$
\left\|y_{\lambda}(t)\right\| \leq \epsilon^{\prime}\left\|z_{\lambda}(t)\right\| \quad \text { for all } t \in\left[t^{*}, t^{\prime}\right)
$$

Since $k(\cdot) \notin L_{\infty}\left(0, t^{\prime}\right)$, it follows that $\lim _{t \rightarrow t^{\prime}} \lambda(t)=0$, and therefore

$$
\dot{v}(t)=\left[A_{4}+(\lambda(t)+\dot{\lambda}(t) t) I_{n-m}\right] v(t)
$$

is exponentially stable, i.e. there exist $M, \mu>0$ such that

$$
\|v(t)\| \leq M e^{-\mu\left(t-t_{0}\right)}\left\|v\left(t_{0}\right)\right\| \text { for all } t_{0} \in\left[0, t^{\prime}\right), t \in\left[t_{0}, t^{\prime}\right)
$$

Using variation of constants for (3.6b) and applying (2.5),(3.15) and (3.16) yields for all $t^{\prime} \geq t \geq t_{0} \geq t^{*}$

$$
\begin{gathered}
\left\|z_{\lambda}(t)\right\| \leq M e^{-\mu\left(t-t_{0}\right)}\left\|z_{\lambda}\left(t_{0}\right)\right\|+\left\|A_{3}\right\| M \int_{t_{0}}^{t} e^{-\mu(t-s)}\left\|y_{\lambda}(s)\right\| d s \\
+\hat{g}\|S\| M \int_{t_{0}}^{t} e^{-\mu(t-s)}\left[\left\|y_{\lambda}(s)\right\|+\left\|z_{\lambda}(s)\right\|\right] d s \\
\leq M e^{-\mu\left(t-t_{0}\right)}\left\|z_{\lambda}\left(t_{0}\right)\right\|+M\left[\left\|A_{3}\right\| \epsilon^{\prime}\right. \\
\left.+\hat{g}\|S\|\left(1+\epsilon^{\prime}\right)\right] \int_{t_{0}}^{t} e^{-\mu(t-s)}\left\|z_{\lambda}(s)\right\| d s
\end{gathered}
$$

Since

$$
L_{t_{0}}: L_{p}\left(t_{0}, t^{\prime}\right) \rightarrow L_{p}\left(t_{0}, t^{\prime}\right), v(\cdot) \mapsto\left(t \mapsto \int_{t_{0}}^{t} e^{-\mu(t-s)}\|v(s)\| d s\right)
$$


is well defined and $\left\|L_{t_{0}}\right\|_{L_{p}\left(t_{0}, t^{\prime}\right)} \leq\left\|L_{0}\right\|_{L_{p}(0, \infty)}=: L<\infty$ (see Vidyasagar 1978, p.250), taking $L_{p}$-norms in (3.17) yields for some $K>0$ and all $t \in\left[t^{*}, t^{\prime}\right)$

$$
\left\|z_{\lambda}(\cdot)\right\|_{L_{p}\left(t^{*}, t\right)} \leq M K\left\|z_{\lambda}\left(t_{0}\right)\right\|+M\left[\left\|A_{3}\right\| \epsilon^{\prime}+\hat{g}\|S\|\left(1+\epsilon^{\prime}\right)\right] L\left\|z_{\lambda}(\cdot)\right\|_{L_{p}\left(t^{*}, t\right)}
$$

Now for $\epsilon^{\prime}$ and $\hat{g}$ small enough it follows from (3.18) that $z_{\lambda}(\cdot) \in L_{p}\left(0, t^{\prime}\right)$. Consequently $y_{\lambda}(\cdot) \in L_{p}\left(0, t^{\prime}\right)$ and hence $k(\cdot) \in L_{\infty}\left(0, t^{\prime}\right)$ which is impossible by assumption. This completes the proof.

It is now possible to prove a new adaptive (exponential) stabilization result incorporating the defined switching strategy (3.5) and the $L_{p}$-based adaptive gain $k(t)$ and exponential weighting.

ThEOREM 3.3. Suppose $p \geq 1, \omega(\cdot): \mathbb{R}_{+} \rightarrow \mathbb{R}_{+}$is continuously differentiable and satisfies (3.1), $1<S_{1}<S_{2}<\ldots$ is a sequence of 'thresholds' with $\lim _{i \rightarrow \infty} S_{i}=$ $\infty$, and $k(\cdot)$ satisfies (3.2),(3.3). If $(A, B, C) \in \Sigma$ is subject to the disturbances (1.8),(1.9) and if the linear bound $\hat{g}$ of $g$ is sufficiently small, then the feedback law

$$
u(t)=S(t) k(t) y(t), \quad S(\cdot) \text { defined in (3.5) }
$$

applied to (1.10) yields:

(i) the absolutely continuous solution of the closed-loop system (1.10), (3.2), (3.19) exists on $\mathbb{R}_{+}$;

(ii) $\lim _{t \rightarrow \infty} k(t)=k_{\infty}<\infty \quad$ and, if $\omega(\cdot) \not \equiv 0$, then $\lim _{t \rightarrow \infty}(\omega \circ k)(t)=\omega_{\infty}>0$;

(iii) $y_{\omega \circ k}(\cdot), y_{\omega_{\infty}}(\cdot) \in L_{p}(0, \infty)$;

(iv) if $\omega(\cdot) \not \equiv 0$, then there exist $M, \mu>0$ such that

$$
\|x(t)\| \leq M e^{-\mu t} \quad \text { for all } t \geq 0,
$$

if $\omega(\cdot) \equiv 0$, then $\lim _{t \rightarrow \infty} x(t)=0 ;$

(v) only a finite numbers of switches occur and $\lim _{t \rightarrow \infty} \varphi(t)=\varphi_{\infty}<\infty$.

Proof. Due to the discontinuity of $S$ and to the nonlinearity of the right hand side of the closed-loop system $(1.10),(3.2),(3.19),(3.5)$, the proof requires a certain number of technicalities. For brevity, we omit in the following proof to take into account the discontinuity of $S$. This can be done in a similar manner as in Ilchmann and Logemann (1991).

Let $\left[0, t^{\prime}\right), t^{\prime} \in(0, \infty]$, be the maximal interval of the unique solution $x(\cdot)$ of (1.10), (3.2),(3.19) and suppose $P>0$ satisfies (3.8).

(a) We first prove that $k(\cdot) \in L_{\infty}\left(0, t^{\prime}\right)$. Suppose otherwise, then by (3.1) $\lim _{t \rightarrow t^{\prime}}(\omega \circ k)(t)=0$ and Proposition 2.3 yields for some $M_{1}>0$ and all $t \in\left[0, t^{\prime}\right)$

$$
\begin{aligned}
& \frac{1}{p}\left\|y_{\omega \circ k}(t)\right\|_{P}^{p} \leq M_{1}+M_{1} \int_{0}^{t}\left\|y_{\omega \circ k}(s)\right\|_{P}^{p} d s \\
& \quad+\frac{1}{2} \operatorname{sgn}(C B) \int_{0}^{t} S(\tau) k(\tau)\left\|y_{\omega \circ k}(\tau)\right\|_{P}^{p-1}\left\|y_{\omega \circ k}(\tau)\right\| \cdot\left\|\beta\left(y_{\omega \circ k}(\tau)\right)\right\| d \tau
\end{aligned}
$$


or, equivalently,

$$
\frac{1}{p}\left\|y_{\omega \circ k}(t)\right\|_{P}^{p} \leq M_{1}+\int_{0}^{t}\left\|y_{\omega \circ k}(s)\right\|^{p} d s\left[M_{1}+\frac{1}{2} \operatorname{sgn}(C B) \varphi(t)\right]
$$

It follows from $(3.21)$ that $\varphi(\cdot) \in L_{\infty}\left(0, t^{\prime}\right)$ since otherwise the right hand side of (3.21) takes both positive and negative values, contradicting (3.21). As $\varphi(\cdot)$ is bounded and monotonic on each interval $\left[t_{i}, t_{i+1}\right)$ it has a finite limit $\lim _{t \rightarrow t^{\prime}} \varphi(t)=$ $\varphi_{\infty}^{\prime}$ and hence a finite number of switching times $t_{1}<\ldots<t_{M}<t^{\prime}$ occur. In particular, $\left.S(\cdot)\right|_{\left[t_{M}, t^{\prime}\right)}$ is constant with value +1 or -1 .

If $S(\cdot) \mathfrak{|}_{\left[t_{M}, t^{\prime}\right)} \equiv-\operatorname{sgn}(C B)$, then $(3.20)$ yields for some $M_{2}$ and $M_{3}$

$$
\frac{1}{p}\left\|y_{\omega \circ k}(t)\right\|_{P}^{p} \leq M_{1}+M_{2} \int_{0}^{t}\left\|y_{\omega o k}(s)\right\|^{p} d s-M_{3} \int_{0}^{t} k(s)\left\|y_{\omega_{0} k}(s)\right\|^{p} d s
$$

Choose $\bar{t} \in\left(0, t^{\prime}\right)$ sufficiently large so that $M_{2}-M_{3} k(\bar{t})<0$. Then it follows from (3.22) that

$$
\begin{aligned}
\frac{1}{p}\left\|y_{\omega \circ k}(t)\right\|_{P}^{p} \leq & M_{1}+\int_{0}^{\bar{t}}\left[M_{2}-M_{3} k(s)\right]\left\|y_{\omega \circ k}(s)\right\|^{p} d s \\
& +\left[M_{2}-M_{3} k(\bar{t})\right] \int_{\bar{t}}^{t}\left\|y_{\omega \circ k}(s)\right\|^{p} d s
\end{aligned}
$$

If $y_{\omega^{\circ} k}(\cdot) \notin L_{p}\left(0, t^{\prime}\right)$, then the right hand side (3.23) becomes negative which is a contradiction. Therefore $y_{\omega \circ k}(\cdot) \in L_{p}\left(0, t^{\prime}\right)$ and $(3.23)$ yields $y_{\omega \circ k}(\cdot) \in L_{\infty}\left(0, t^{\prime}\right)$.

It remains to consider the case $\left.S(\cdot)\right|_{\left[t_{M}, t^{\prime}\right)} \equiv \operatorname{sgn}(C B)$. Suppose the system is of the form (3.6) with $u(t)=\operatorname{sgn}(C B) k(t) y(t)$ and $\lim _{t \rightarrow t^{\prime}} k(t)=\infty$. Then, for $\epsilon>0$, by Lemma 3.3 there exists a $t_{\epsilon} \in\left(t_{M}, t^{\prime}\right)$ such that for all $t \in\left(t_{\epsilon}, t^{\prime}\right) \backslash J, J$ defined in $(3.11)$, we obtain

$$
\begin{aligned}
\frac{1}{p}\left(\frac{d}{d t}\left\|y_{\omega \circ k}(t)\right\|_{P}^{p}\right)= & \left\|y_{\omega \circ k}(t)\right\|_{P}^{p-1}\left\langle\beta\left(y_{\omega \circ k}(t)\right), P A_{1} y_{\omega \circ k}(t)+P A_{2} z_{\omega 0 k}(t)\right. \\
& \left.\quad+\operatorname{sgn}(C B) k(t) P C B y_{\omega \circ k}(t)+P\left[g^{1}(t)+C B h_{\omega 0 k}(t)\right]\right\rangle \\
\geq & \left\|y_{\omega 0 k}(t)\right\|_{P}^{p} M_{4}\left[M_{5}-\epsilon+\frac{1}{2} k(t)\right]
\end{aligned}
$$

for suitable choice of $M_{4}, M_{5} \geq 0$. This proves arbitrary fast exponential growth of $y(\cdot)$ on $\left[t_{\epsilon}, t^{\prime}\right)$. However, this contradicts boundedness of $\varphi(\cdot)$ and so $k(\cdot) \in L_{\infty}\left(0, t^{\prime}\right)$.

(b) Since $g$ and $h$ are linearly bounded on $x$, and since $k(\cdot)$ is bounded on $\left[0, t^{\prime}\right)$ it follows, from the theory of ordinary differential equations, that $t^{\prime}=\infty$ (and so $x(\cdot)$ does not have a finite escape time). Assertions (i)-(iii) and (v) follow immediately.

(c) We assume that (1.10), (3.19) is of the form (3.6). Assertion (iv) is proved only for the case $\omega(\cdot) \not \equiv 0$ (the case $\omega(\cdot) \equiv 0$ is simpler and uses the same argument). We know that $y_{\mu}(\cdot) \in L_{p}(0, \infty)$ for all $\mu \in\left[0, \omega_{\infty}\right]$. Consider (3.6) and choose $\mu, \hat{g}>0$ small enough so that $(3.6 \mathrm{~b})$ yields $z_{\mu}(\cdot) \in L_{p}(0, \infty)$, this can be shown by 
using a similar techniques as in Vidyasagar (1978), pp.258. Since $k(\cdot)$ is bounded and $g$ and $h$ are linearly bounded, it follows from $(3.6)$ that $\dot{x}_{\mu}(\cdot) \in L_{p}(0, \infty)$. Now $x_{\mu}(\cdot), \dot{x}_{\mu}(\cdot) \in L_{p}(0, \infty)$ implies $\lim _{t \rightarrow \infty} x_{\mu}(t)=0$, which completes the proof.

\section{REMARK 3.4 .}

(i) Note that the gain adaptation $y \mapsto k$ is achieved using an exponentially weighted output and hence involves multiplication by an unbounded function if $\omega(\cdot) \not \equiv 0$. If $\omega(\cdot) \equiv 0$ in Theorem 3.4 the results still hold true with the modification that only asymptotic stabilization of $x(t)$ is ensured. The unboundedness property is avoided in this case at the expense of guaranteed exponential stabilization.

(ii) If only asymptotic stabilization is of interest and we put $\omega(\cdot) \equiv 0$, then the adaptive stabilizer $(3.5),(3.19)$ rejects every disturbance $d(\cdot) \in L_{p}(0, \infty)$ incorporated into the system state equation as follows

$$
\dot{x}(t)=A x(t)+g(t, x(t))+d(t)+B[u(t)+h(t, x(t))]
$$

For brevity a proof of this is omitted.

\section{Robustness with respect to input-output nonlinearities}

In the single-input single-output case, a suitable extension of the switching strategy presented above can also tolerate arbitrary sector bounded actuator and sensor nonlinearities as defined (1.11). This has been considered by Logemann and Owens (1988) using a Nussbaum based switching strategy and without taking into account the nonlinearities $g$ and $h$. However it is necessary to find a different proof in this case. Only sector-bounded actuator (not sensor) nonlinearities have been considered in Ilchmann and Owens (1991a), without allowing the inclusion of $g$ and $h$ perturbations to the systems dynamics.

If we allow sector-bounded actuator and sensor nonlinearities, then only $\hat{y}(t)=$ $\eta(t, y(t))$ and $\hat{u}(t)=-S(t) k(t) \hat{y}(t)$ are available but not $y(t)$ and $u(t)=\xi(t, \hat{u}(t))$. We have to introduce certain indicator functions to act as the basis for switching decisions in the absence of information on $y(t)$ and precise knowledge of what $u(t)$ will be as a consequence of our decision. For $\omega(\cdot)$ and $k(\cdot)$ as defined in (3.1)-(3.3), the switching decision function proposed is

$$
\psi(t):= \begin{cases}k(0), & \text { if } \quad \int_{0}^{t}\|\hat{y}(\tau)\|^{p} d \tau=0 \\ \int_{0}^{t} S(\tau) k(\tau)\left\|\hat{y}_{\omega \circ k}(\tau)\right\|^{p} d \tau / \int_{0}^{t}\left\|\hat{y}_{\omega \circ k}(\tau)\right\|^{p} d \tau, & \text { if } \quad \int_{0}^{t}\|\hat{y}(\tau)\|^{p} d \tau \neq 0\end{cases}
$$

(which contains only measurables) and the thresholds will be replaced by quantities related to the function

$$
\phi(t):= \begin{cases}k(0), & \text { if } \quad \int_{0}^{t}\|\hat{y}(\tau)\|^{p} d \tau=0 \\ \int_{0}^{t} k(\tau)\left\|\hat{y}_{\omega \circ k}(\tau)\right\|^{p} d \tau / \int_{0}^{t}\left\|\hat{y}_{\omega \circ k}(\tau)\right\|^{p} d \tau, & \text { if } \quad \int_{0}^{t}\|\hat{y}(\tau)\|^{p} d \tau \neq 0\end{cases}
$$


Clearly, $\phi(t)$ is positive and non-decreasing since $k(t)$ is positive and non-decreasing. Moreover

$$
\zeta(t):=\frac{\psi(t)}{\phi(t)} \in[-1,1]
$$

Let $t_{0}>0$ be such that $0<\left|\psi\left(t_{0}\right)\right|<\phi\left(t_{0}\right)$ and consequently

$$
\zeta(t) \in(-1,1) \text { for all } t \geq t_{0} .
$$

This can always be achieved by application of a random sequence of switches in an interval $0<t<t_{0}$. Note that $\zeta(t)$ is monotonic on any interval where $S(t)$ is constant. The suggested switching algorithm for $t \geq t_{0}$ is as follows: after the initialization phase of random switching,

$$
\left.\begin{array}{ll} 
& i:=0 \quad, S\left(t_{0}\right):=1 \\
& t_{i+1}:=\inf \left\{t>t_{i} \mid S\left(t_{i}\right) \zeta(t)=1-\hat{S}_{i+1}\right\} \\
& S(t):=S\left(t_{i}\right) \text { for all } t \in\left[t_{i}, t_{i+1}\right) \\
& S\left(t_{i+1}\right):=-S\left(t_{i}\right) \\
& i:=i+1 \\
& \text { go to }(*)
\end{array}\right\}
$$

where $1-\zeta\left(t_{0}\right)>\hat{S}_{1}>\hat{S}_{2}>\ldots>0$ is a sequence converging to zero. Note that, with $\eta(\cdot, y) \equiv y$, the algorithm (4.3) is that of (3.5) with adaptive thresholds $S_{i}=k\left(t_{0}\right)^{-1}\left(1-\hat{S}_{i}\right)$ and $\zeta(t)=\varphi(t)$. It is based on measured quantities only. The technical problem is that these quantities do not have a precise known relation to the inputs and outputs of the (nonlinearly perturbed) internal linear part of the plant. In the following useful bounds and relationships between the important quantities are derived.

The input-output nonlinearities are assumed to be sector bounded, i.e. $\xi(\cdot, \cdot) \in$ $\mathcal{S B}(\alpha, \beta)$, for some $\beta \geq \alpha>0$, and $\eta(\cdot, \cdot) \in \mathcal{S B}\left(\alpha^{\prime}, \beta^{\prime}\right)$, for some $\beta^{\prime} \geq \alpha^{\prime}>0$, see (1.11). Introducing the notation

$$
\begin{aligned}
& \bar{\alpha}:=\frac{\alpha}{\left(\beta^{\prime}\right)^{p-1}}, \quad \text { and } \quad \bar{\beta}:=\frac{\beta}{\left(\alpha^{\prime}\right)^{p-1}} \\
& \bar{S}(t):=\left\{\begin{array}{rll}
-\bar{\beta} & \text { if } & S(t)=+1 \\
\bar{\alpha} & \text { if } & S(t)=-1
\end{array}\right. \\
& \underline{S}(t):=\left\{\begin{array}{rll}
-\bar{\alpha} & \text { if } & S(t)=+1 \\
\bar{\beta} & \text { if } & S(t)=-1
\end{array}\right.
\end{aligned}
$$

then, for $k(t)$ positive, it is easily checked that for $y(t) \neq 0$

$$
\underline{S}(t) k(t)|\hat{y}(t)|^{p} \leq \xi(t, S(t) k(t) \hat{y}(t)) \cdot y(t)|y(t)|^{p-2} \leq \bar{S}(t) k(t)|\hat{y}(t)|^{p}
$$

and furthermore

$$
\bar{\psi}(t):=-\frac{\int_{0}^{t} \bar{S}(\tau) k(\tau)\left|\hat{y}_{\omega}(\tau)\right|^{p} d \tau}{\int_{0}^{t}\left|\hat{y}_{\omega}(\tau)\right|^{p} d \tau}=\frac{\bar{\beta}-\bar{\alpha}}{2} \phi(t)+\frac{\bar{\beta}+\bar{\alpha}}{2} \psi(t)
$$


with

$$
\underline{\psi}(t):=-\frac{\int_{0}^{t} \underline{S}(\tau) k(\tau)\left|\hat{y}_{\omega}(\tau)\right|^{p} d \tau}{\int_{0}^{t}\left|\hat{y}_{\omega}(\tau)\right|^{p} d \tau}=-\frac{\bar{\beta}-\bar{\alpha}}{2} \phi(t)+\frac{\bar{\beta}+\bar{\alpha}}{2} \psi(t)
$$

Since $\bar{\alpha} \leq \bar{\beta}$, it follows that

$$
\underline{\psi}(t) \leq \frac{\bar{\beta}+\bar{\alpha}}{2} \psi(t) \leq \bar{\psi}(t)
$$

and

$$
\underline{\psi}(t)=\frac{\bar{\beta}+\bar{\alpha}}{2}\left[-\frac{\bar{\beta}-\bar{\alpha}}{\bar{\beta}+\bar{\alpha}}+\zeta(t)\right] \phi(t), \quad \text { and } \quad \bar{\psi}(t)=\frac{\bar{\beta}+\bar{\alpha}}{2}\left[\frac{\bar{\beta}-\bar{\alpha}}{\bar{\beta}+\bar{\alpha}}+\zeta(t)\right] \phi(t)
$$

This sets us in a position to prove the following lemma which is crucial for the main result of this section.

LEMma 4.1. Suppose $p \geq 1, \beta \geq \alpha>0, \beta^{\prime} \geq \alpha^{\prime}>0, t^{\prime}>0, k(\cdot):\left[0, t^{\prime}\right) \rightarrow$ $\mathbb{R}_{+}$is absolutely continuous and $\omega(\cdot)$ satisfies (3.1). Let the single-input singleoutput system $(A, b, c) \in \Sigma$ be subjected to the disturbances $(1.8),(1.9), \xi(\cdot, \cdot) \in$ $\mathcal{S B}(\alpha, \beta), \eta(\cdot, \cdot) \in \mathcal{S B}\left(\alpha^{\prime}, \beta^{\prime}\right)$. Apply the feedback law

$$
\hat{u}(t)=S(t) k(t) \eta(t, y(t))
$$

to (1.1D) where $S(\cdot)$ is a switching function defined via the algorithm (4.3) and its initialization phase on $\left[0, t_{0}\right]$ with $t_{0}>0$ to ensure that $\zeta\left(t_{0}\right) \in(-1,1)$. If the solution of (1.12), (4.9) exists on $\left[0, t^{\prime}\right)$ for some $t^{\prime} \in[0, \infty)$ and $\lim _{t \rightarrow t^{\prime}} k(t)=\infty$, then

(i) $\phi(\cdot), \underline{\psi}(\cdot), \bar{\psi}(\cdot), \psi(\cdot) \in L_{\infty}\left(0, t^{\prime}\right)$

(ii) $y_{w o k}(\cdot) \in L_{i}\left(0, t^{\prime}\right)$ for all $i \in[p, \infty]$.

Proof. Suppose $\phi(\cdot) \notin L_{\infty}\left(0, t^{\prime}\right)$.

(a) If the switching algorithm (4.3) switches an infinite number of times $0=$ $t_{0}<t_{1}<\ldots<t^{\prime}$, then by construction this implies

$$
\limsup _{t \rightarrow t^{\prime}} \zeta(t)=+1 \text { and } \quad \liminf _{t \rightarrow t^{\prime}} \zeta(t)=-1
$$

From (4.8) this gives

$$
\limsup _{t \rightarrow t^{\prime}} \underline{\psi}(t)=\infty \quad \text { and } \quad \liminf _{t \rightarrow t^{\prime}} \vec{\psi}(t)=-\infty
$$

As $k(\cdot)$ is unbounded $\lim _{t \rightarrow t^{\prime}}(\omega \circ k)(t)=0$, and applying (4.9) to Proposition 2.3 yields for some $M>0$ and $\beta$ as defined in (2.6)

$$
\begin{aligned}
\frac{1}{p}\left|y_{\omega \circ k}(t)\right|^{p} & \leq M+M \int_{0}^{t}\left|y_{\omega \circ k}(s)\right|^{p} d s \\
& +c b \int_{0}^{t}\left|y_{\omega \circ k}(s)\right|^{p-1} \beta\left(y_{\omega \circ k}(s)\right) e^{\omega^{\prime} \circ k(s) s} \xi(s, S(s) k(s) \eta(s, y(s)) d s
\end{aligned}
$$


Suppose $c b>0$. Then, by using (4.4),

$$
\frac{c b}{\left(\alpha^{\prime}\right)^{p}} \underline{\psi}(t) \leq \frac{c b \int_{0}^{t} \xi\left(s, S(s) k(s) \eta(s, y(s)) e^{\omega o k(s) s} \beta\left(y_{\omega \circ k}(s)\right)\left|y_{\omega o k}(s)\right|^{p-1} d s\right.}{\int_{0}^{t}\left|y_{\omega \circ k}(s)\right|^{p} d s}
$$

whence

$$
\frac{c b}{\left(\alpha^{\prime}\right)^{P}} \psi(t) \leq \frac{c b}{\left(\beta^{\prime}\right)^{p}} \bar{\psi}(t)
$$

Equation (4.11) together with (4.13) yield that the right hand side of (4.12) takes arbitrary large positive and negative values as $t$ tends to $t^{\prime}$. This contradicts the positiveness of the left hand side and therefore only finitely many switches occur. If $c b<0$ then the definitions of $\bar{S}(\cdot)$ and $\underline{S}(\cdot)$ have to be modified which gives a similar inequality to (4.14) and the identical arguments are valid.

(b) If there is only a finite number of $M$ of switches then

$$
\lim _{t \rightarrow t^{\prime}} \zeta(t)=\zeta_{\infty} \in(-1,+1)
$$

However, the unboundedness of $\phi(\cdot)$ yields the contradiction

$$
\begin{gathered}
\lim _{t \rightarrow t^{\prime}} \zeta(t)=\lim _{t \rightarrow t^{\prime}} \frac{\int_{0}^{t_{M}} S(\tau) k(\tau)\left|\hat{y}_{\omega 0 k}(\tau)\right|^{p} d \tau+S\left(t_{M}\right) \int_{t_{M}}^{t} k(\tau)\left|\hat{y}_{\omega \circ k}(\tau)\right|^{p} d \tau}{\int_{0}^{t_{M}} k(\tau)\left|\hat{y}_{\omega 0 k}(\tau)\right|^{p} d \tau+\int_{t_{M}}^{t} k(\tau)\left|\hat{y}_{\omega 0 k}(\tau)\right|^{p} d \tau} \\
=S\left(t_{M}\right)= \pm 1 .
\end{gathered}
$$

Therefore (a) and (b) prove $\phi(\cdot) \in L_{\infty}\left(, t^{\prime}\right)$.

(c) The boundedness of $\psi(\cdot), \bar{\psi}(\cdot)$, and $\psi(\cdot)$ now follows from (4.8) and (4.7). This proves (i).

(d) For all $T>0$ and $t \in\left(T, t^{\prime}\right)$ we have

$$
\frac{\int_{0}^{T} k(\tau)\left|\hat{y}_{\omega \circ k}(\tau)\right|^{p} d \tau+k(T) \int_{T}^{t}\left|\hat{y}_{\omega \circ k}(\tau)\right|^{p} d \tau}{\int_{0}^{T}\left|\hat{y}_{\omega \circ k}(\tau)\right|^{p} d \tau+\int_{T}^{t}\left|\hat{y}_{\omega \circ k}(\tau)\right|^{p} d \tau} \leq \phi(t)
$$

If $y_{\omega \circ k}(\cdot) \notin L_{p}\left(0, t^{\prime}\right)$ then $\hat{y}_{\omega o k}(\cdot) \notin L_{p}\left(0,, t^{\prime}\right)$ and thus the limit of the left hand side of $(4.15)$ would be $k(T)$. Since $k(T)$ is arbitrary, this contradicts the boundedness of $\phi(\cdot)$. Hence $y_{\omega \circ k}(\cdot) \in L_{p}\left(0, t^{\prime}\right)$.

(e) Since $y_{\omega \circ k}(\cdot) \in L_{p}\left(0, t^{\prime}\right)$, a rough estimate applied to (4.12) yields $y_{\omega \circ k}(\cdot) \in$ $L_{\infty}\left(0, t^{\prime}\right)$. This completes the proof.

THEOREM 4.2. Suppose $p \geq 1, \omega(\cdot): \mathbb{R}_{+} \rightarrow \mathbb{R}_{+}$is continuously differentiable and satisfies (3.1), $k(\cdot)$ satisfies (3.2), (3.3). The switching function $S(\cdot)$ is defined as in algorithm (4.3). If the single-input single-output system (1.12) is considered under the assumptions as given in Lemma 4.1 then the feedback law (4.9) applied to (1.12) yields: 
(i) the solution $x(\cdot)$ exists on $[0, \infty)$;

(ii) $\lim _{t \rightarrow \infty} k(t)=k_{\infty}<\infty, \quad \lim _{t \rightarrow \infty}(\omega \circ k)(t)=\omega_{\infty}>0$, $\lim _{t \rightarrow \infty} \psi(t)=\psi_{\infty} \in \mathbb{R}, \quad \lim _{t \rightarrow \infty} \phi(t)=\phi_{\infty} \in \mathbb{R} ;$

(iii) there are only a finite number of switches;

(iv) $y_{\omega \circ k}(\cdot), y_{\omega_{\infty}}(\cdot) \in L_{p}(0, \infty)$;

(v) if $\omega(\cdot) \not \equiv 0$, then there exist $M, \mu>0$ such that

$$
\|x(t)\| \leq M e^{-\mu t} \quad \text { for all } t \geq 0
$$

if $\omega(\cdot) \equiv 0$, then $\lim _{t \rightarrow \infty} x(t)=0$

Proof. As in Theorem 3.4 we ignore, for brevity, the discontinuity of $S$. Let $\left[0, t^{\prime}\right)$ be the maximal interval of existence of $x(\cdot)$ of (1.12), (4.9).

(a) Suppose $k(\cdot) \notin L_{\infty}\left(0, t^{\prime}\right)$. Then Lemma 4.1 (ii) yields together with (3.3) that $k(\cdot) \in L_{\infty}\left(0, t^{\prime}\right)$. This is a contradiction.

(b) Since $k(\cdot) \in L_{\infty}\left(0, t^{\prime}\right)$, it follows as in part (b) of the proof of Theorem 3.4 that $t^{\prime}=\infty$.

(c) Now (iv) follows from (3.3). This implies that (iii) is valid by definition of $\phi(\cdot)$ and $\psi(\cdot)$.

(d) To prove (ii) denote the intervals where $S(\cdot)$ in constant by $\left[t_{i}, t_{i+1}\right)$. Then, since $\hat{y}_{\omega \circ k}(\cdot) \in L_{p}\left(0, t^{\prime}\right)$,

$$
\lim _{t \rightarrow t^{\prime}} \zeta(t)=\lim _{t \rightarrow \infty} \frac{\sum_{i \geq 0}(-1)^{i} \int_{t_{i}}^{t_{2+1}} k(\tau)\left|\hat{y}_{\omega \circ k}(\tau)\right|^{p} d \tau}{\sum_{i \geq 0} \int_{t_{i}}^{t_{i+1}} k(\tau)\left|\hat{y}_{\omega \circ k}(\tau)\right|^{p} d \tau} \in(-1,1)
$$

and (ii) follows from the switching algorithm.

(e) Assertion (v) can be shown by using similar arguments as in Theorem 3.4. This completes the proof.

The analogous remark as in Remark 3.5 holds for Theorem 4.2.

\section{Simulations}

Consider the system described by the state space model

$$
\begin{aligned}
& \dot{x}(t)=A x(t)+b u(t), \quad x(0)=x_{0} \\
& y(t)=c x(t)
\end{aligned}
$$

where

$$
A=\left[\begin{array}{ccc}
2 & 1 & 1 \\
1 & -1 & 3 / 4 \\
1 & -3 / 4 & -1
\end{array}\right], \quad b=c^{T}=\left[\begin{array}{l}
1 \\
0 \\
0
\end{array}\right], \quad x_{0}=\left[\begin{array}{l}
1 \\
0 \\
1
\end{array}\right]
$$


The system is obviously of relative degree 1 with $c b=1$ and also minimum phase since

$$
\operatorname{det}\left[\begin{array}{cc}
s I-A & b \\
c & 0
\end{array}\right]=(s+1)^{2}+(3 / 4)^{2}
$$

To illustrate the effect of the choice of $p$, we consider the following adaptive stabilizer with $p=1,2,4$

$$
\begin{aligned}
& u(t)=S(t) k(t) y(t) \\
& \dot{k}(t)=|y(t)|^{p}, \quad k(0)=0
\end{aligned}
$$

where $S(\cdot)$ is determined in (3.5) with the modification $S\left(t_{i}\right) \varphi\left(t_{i+1}\right)=\lambda_{i+1}\left[k\left(t_{0}\right)+1\right]$ defining the switching times.

Three different 'threshold sequences' $\left\{\lambda_{i}\right\}$ are considered and no input-output nonlinearities are included. The form of the threshold sequences are indicated on the relevant figures.

Note that the system reacts much faster if $p$ is larger due to the increase in the rate of change of the gain whenever $y(t)$ increases above unity. This has the effect that the transient behaviour of $y(t)$ improves as $p$ becomes larger. More precisely, in Fig.2, the peaking of the output response is removed with increases in $p$, this behaviour being seen also in Figs. 3 and 4 . The effect of $p$ on the limit gains has no pattern other than that the case of $p=1$ leads to in all the cases described to the highest value. In all cases the number of switches is the same as $p$ increases but the switches occur more rapidly and at lower gain values suggesting that the use of 'large' $p$ will tend to reduce peaking phenomena. The general form of the responses seems to be relatively insensitive to the choice of threshold sequence, no real pattern being discerned from these results.

These results are for one initial condition only. Changes in initial conditions show similar results although the number of switches can increase considerably. 


\section{Gain functions}
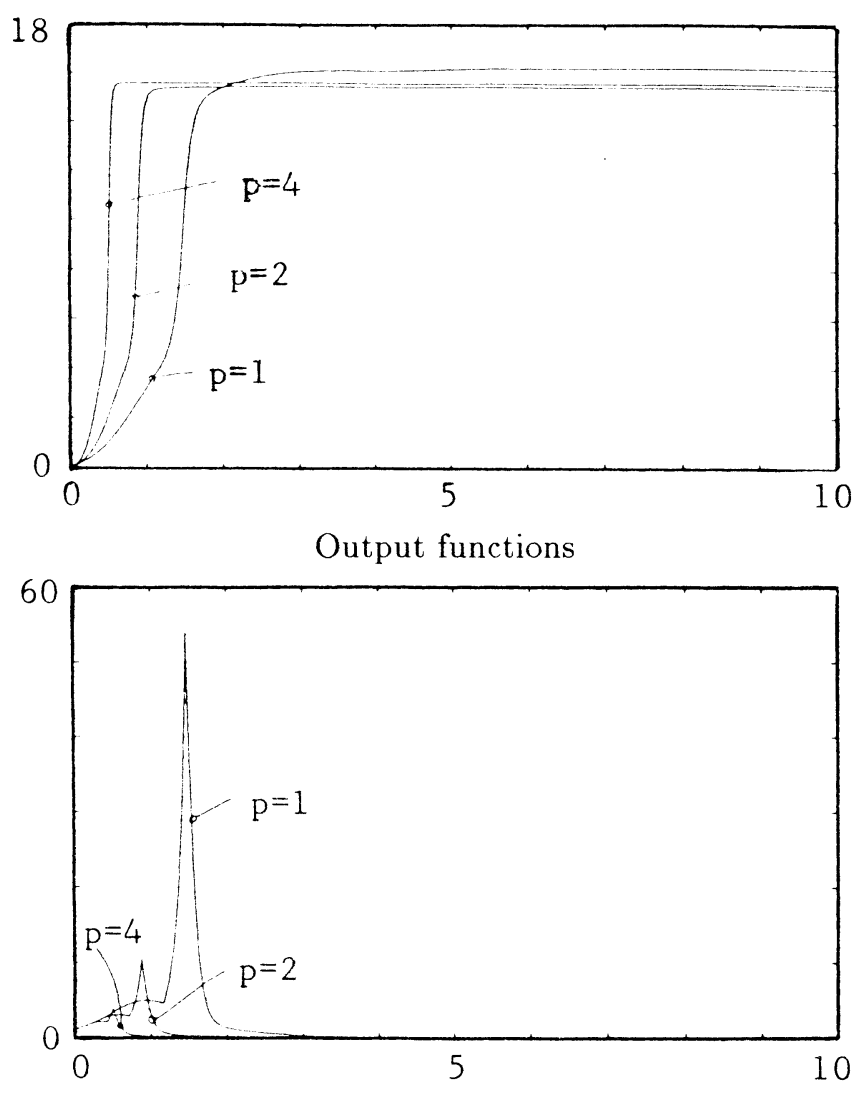

Sign and switching decision functions

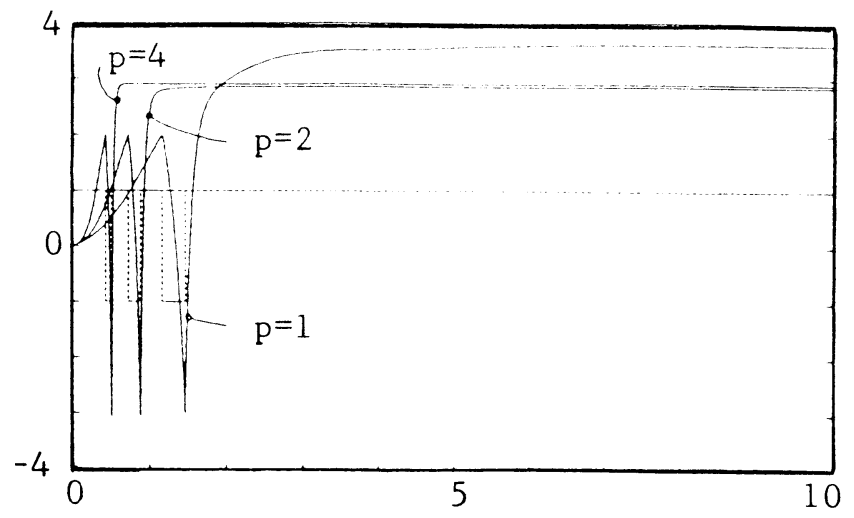

FIG. 2. $\quad \lambda_{i}=i+1$ 
Gain functions
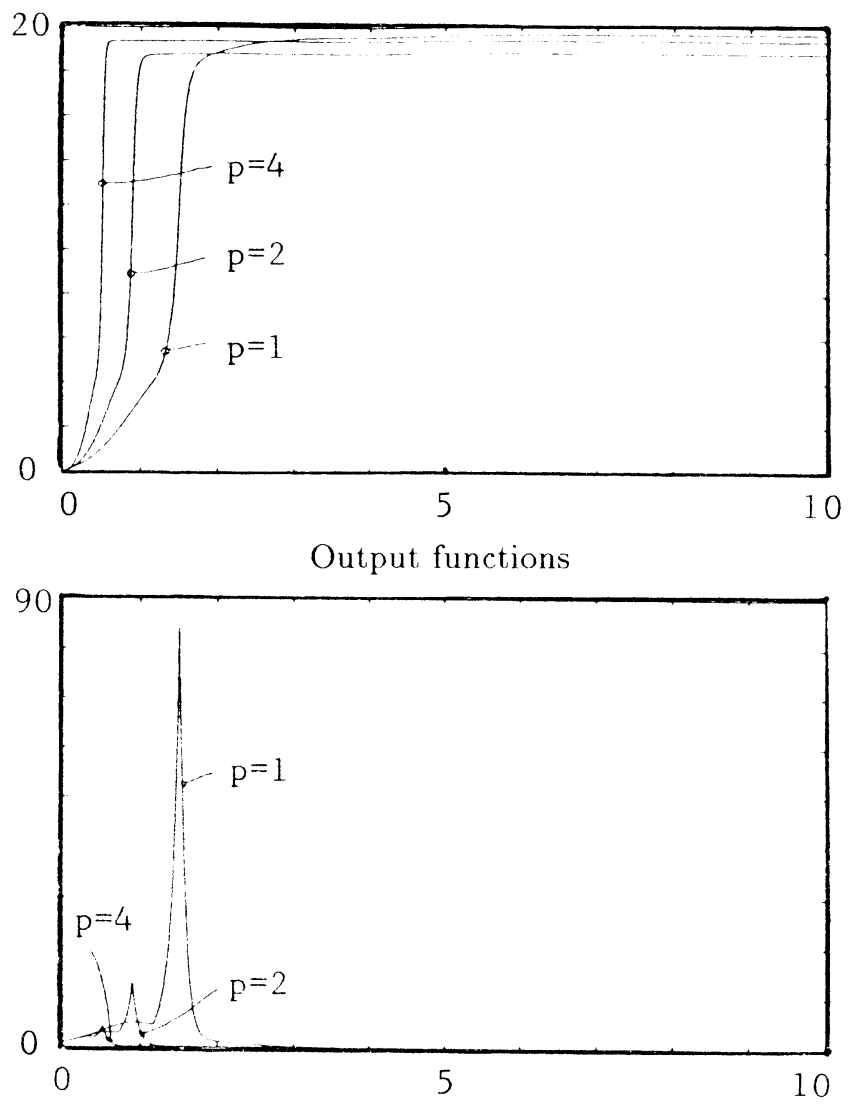

Sign and switching decision functions

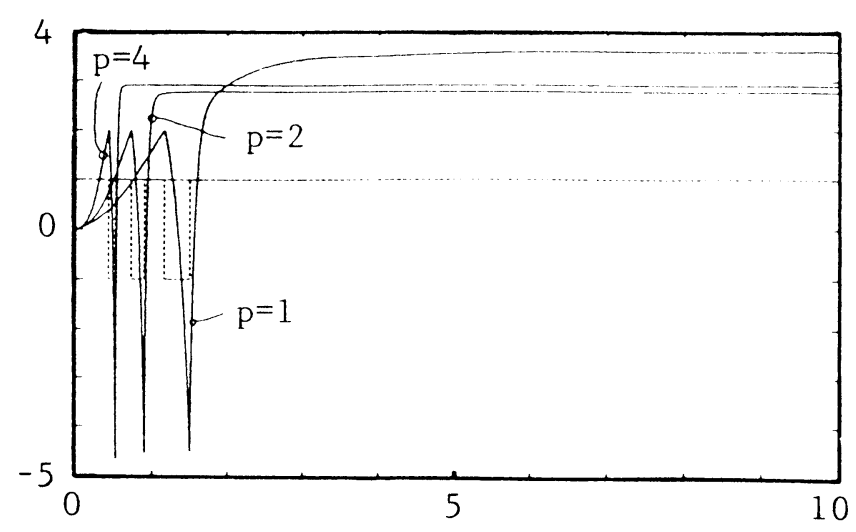

FIG. 3. $\quad \lambda_{i}=\frac{1}{2}(i+2)^{2}$ 


\section{Gain functions}
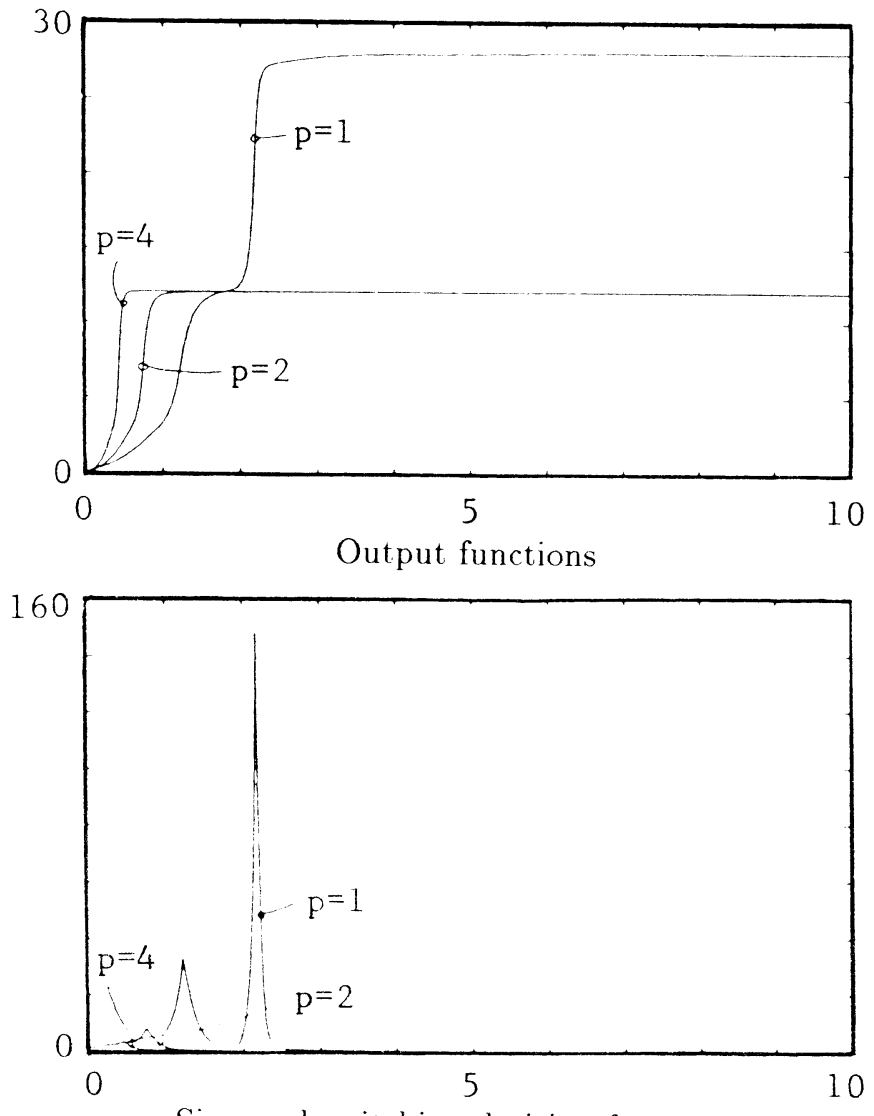

Sign and switching decision functions

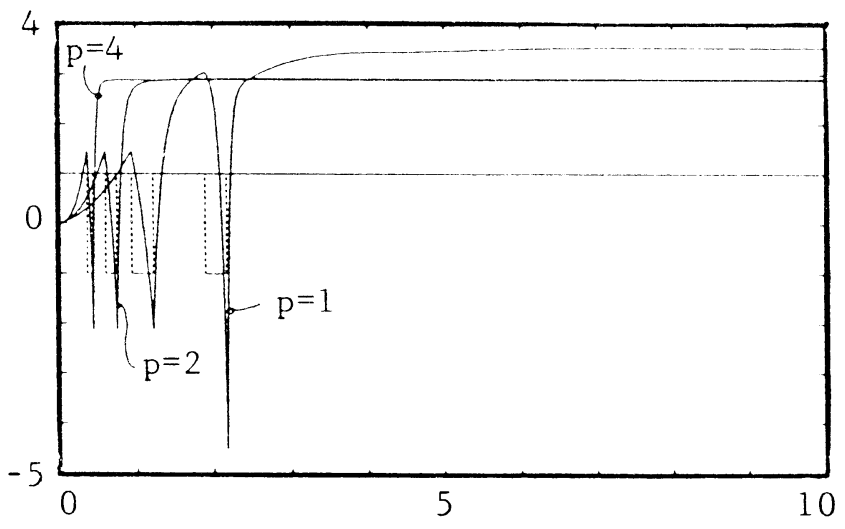

FIG. 4. $\quad \lambda_{i}=(1.45)^{i}$ 


\section{Acknowledgement}

We are indebted to ERASMUS student Minne Cuperus of the University of Twente for carefully performing the simulations presented in Section 5.

The work was supported by the EEC SCIENCE programme under grant number SC-1-0433-C- $(\Lambda)$ and by the University of Exeter. The main support for the first author came from the German Research Association (DFG).

\section{REFERFNCES}

Brrnes, C.I. \& Willams, J.C. 1984 Adaptive stabilization of multivariable linear systems. Proc. 23rd Conf. on Decision and Control, Las Vegas, pages 1574-1577. Ilchmann, A. \& Logemann, H. 1991 High-gain adaptive stabilization of multivariable linear systems - revisited. Report 242, Institut für Dynamische Systeme, Universität Bremen, to appear in Systems \& Control Letters.

Ilchmann, A.\& Owens, I).H. 1990 Adaptive stabilization with exponential decay. Systems 8 Control Letters, 14, 437-443.

ILCHMANN, A. \& OWENS, D.H. 1991 Exponential stabilization using non-differential gain adaptation. IMA Journal of Math. Control \& Information, 7, 339-349.

IlCHMAnN, A. \&. OWEns, D.H. 1991 a Robust universal adaptive stabilization in the presence of nonlinearities. Proc. 1st European Control Conference, Grenoble, pages $2580-2585$.

Iichmann, $\Lambda$., Owens, D.H. \& Prätzel-Wolters, D. 1987 High gain robust adaptive controllers for multivariable systems. Systems Control Letters, 8, 397404.

LOGEMANN, H. \& OWENS, D.H. 1988 Input-output theory of high -gain adaptive stabilization of infinite dimensional systems with non-linearities. Int. J. Adaptive Control \& Signal Processing, 2, 193-216.

Märlensson, B. 1986 Adaptine Stabilization. PhD Thesis, Itund Institute of 'Technology, Lund, Sweden.

Miluer, D.E. \& Davison, E.J. 1989 An adaptive controller which provides Lyapunov stability. IEEE Trans. Automatic Control, AC-34, 599-609.

Morse, A.S. 1987 High gain feedback algorithms for adaptive stabilization. Proc. 5th Yale Workshop on Applcations of Adaptive Systems Theory, pages 13-18.

Nussbaum, R.D. 1983 Some remarks on a conjecture in parameter adaptive control. Systems \& Control Letters, 3, 243-246.

Owens, D.H., Prätzhl-Wolters, D. \& Ilchmann, A. 1987 Positive-real structure and high-gain adaptive stabilization. IMA J. of Math. Control 8 Information. 4, $167-181$.

Vinyasagar, M. 1978 Nonlincar Systems Analysis, Prentice Hall, Fnglewood Cliffs.

Willems, J.C. \& Byrnes, C.I. 1984 Global adaptive stabilization in the absence of information on the sign of the high frequency gain. Lect. Notes Control 6 Information Sciences, 62, pages 49-57, Springer-Verlag, Berlin. 